\title{
Diabetische Nephropathie - Therapie
}

\section{Diätetische Maßnahmen}

Für Patienten mit diabetischer Nephropathie gelten vor allem in frühen Stadien die gleichen Empfehlungen wie für Diabetiker ohne Nephropathie. Auf eine ausreichende Kalorienzufuhr ist zu achten, strenge Reduktionsdiäten sind kontraindiziert. Ein BMI (Body Mass Index) unterhalb der 50. Perzentile ist ein unabhängiger Prädiktor für Mortalität (1). Dies gilt v.a. für ältere Patienten, die häufig eine Malnutrition aufweisen. Der Serumalbuminspiegel gilt als Marker für den Ernährungszustand und die klinische Relevanz eines Eiweißverlustes. Zur Beurteilung einer Malnutrition können der BMI, der Muskelquerschnitt des M. Rectus femoris sowie weitere Laborparameter (CRP, Lymphozytenzahl, Transferrin, Lipide) herangezogen werden.

Eine vermehrte Eiweißzufuhr erhöht möglicherweise den intraglomerulärenDruck. Eine EinschränkungderEiweißzufuhrführtbeiTyp1-Diabetikern zu einem Rückgang der Proteinurie und im Trend zu einer Verlangsamung der Progression der Niereninsuffizienz. Eine Zufuhrvonetwa 0,8 gEiweißprokgKörpergewicht(KG)proTaggiltzwar als Richtwert, jedoch könnengeringere MengenEiweißschnell zuProteinmalnutrition führen (2). Bei präterminal eingeschränkter Nierenfunktion (glomeruläre Filtrationsrate (GFR) $<25 \mathrm{ml} / \mathrm{Tag}$ ) wird eine weitere Senkung der Eiweißzufuhr auf etwa 0,6 g / kg KG / Tag diskutiert. In der Praxis wird dies jedoch von den Patienten selten akzeptiert und ist oft wegen einer begleitenden Malnutrition oder renaler Eiweißverluste auch nicht indiziert. Da Fasten bei urämischen Patienten eine stark katabole Stoffwechselsituation hervorrufen kann, sollte auf eine starke Gewichtsreduktion verzichtet werden. Im Stadium der dialysepflichtigen Niereninsuffizienz dagegen ist unbedingt eine erhöhteEiweißzufuhrvon 1,2 g/ kg KG/Tag zu empfehlen. Die Hälfte desEiweißbedarfs sollteaus tierischenProduktengedecktwerden.

\section{Blutzuckertherapie}

Für den Typ-1-Diabetes konnte in der DCC-Studie der Effekt einer intensivierten Insulintherapie auf das Fortschreiten einer Nephropathie gezeigt werden. In der Primärpräventionsgruppe (bis 5 Jahre Diabetesdauer) wurde das kumulative Risiko für eine Mikroalbuminurie um 34\%, in der Sekundärpräventionsgruppe um $43 \%$ pro Jahr gesenkt (3). Bei Patienten mit Typ-2-Diabetes verzögert eine intensivierte Glukosekontrolle über 10 Jahre mit Sulfonylharnstoffen oder Metformin oder Insulin das Auftreten und die Progression einer Mikroalbuminurie um insgesamt $11 \%$, hat jedoch keinen signifikanten Einfluss auf die Nierenfunktion (4). Die nicht signifikanten Ergebnisse weiterer prospektiver Studien können möglicherweise auch auf die relativ kurzen Verlaufszeiten zurückgeführt werden. Die medikamentöse Therapie des Diabetes erfolgt bei fortgeschrittener Nephropathie meist mit Insulin, da Metformin und Sulfonylharnstoffe (Ausnahme: Gliquidon) kontraindiziert sind. Bei der Bestimmung des $\mathrm{HbA}_{1 \mathrm{c}}$ Wertes ist zu beachten, dass durch die Carbamylierung des Hämoglobins die Normwerte des $\mathrm{HbA}_{1 \mathrm{c}}$ Wertes gering angehoben sind.

lkurzgefasst: Für Patienten mit diabetischer Nephropathie gelten insbesondere in frühen Stadien die gleichen diätetischen Empfehlungen wie für Diabetiker ohne Nephropathie. Besondere Aufmerksamkeit gilt der Eiweißzufuhr, die dem Stadium der Niereninsuffizienz und dem Ernährungszustand angepasst werden sollte. Die medikamentöse Therapie des Diabetes erfolgt bei fortgeschrittener Nephropathie meist mit Insulin, da Metformin und Sulfonylharnstoffe (Ausnahme: Gliquidon) kontraindiziert sind.

${ }^{1}$ Heinrich Heine Universität, Klinik für Nephrologie und Rheumatologie (Direktor: Prof. Dr. Grabensee), Düsseldorf

${ }^{2}$ Kliniken St. Antonius, Wuppertal 


\section{Antihypertensive Therapie}

Eine arterielle Hypertonie wurde als eigenständiger Risikofaktor für die Entwicklung einer chronischen Niereninsuffizienz identifiziert (5), ist aber auch Folge der diabetischen Nephropathie (6). Als Ursache werden u.a. eine Erhöhung des intraglomerulären Drucks, die Hyperfiltration von Makromolekülen, die Proliferation von Mesangialzellen, eine Endothelschädigung und die Expansion und Modifikation der extrazelluären Matrix diskutiert.

Zahlreiche Studien (HOT (8), Syst-Eur, SHEP, UKPDS (12), CAPPP, $A B C D, H O P E(11))$ haben gezeigt, dass eine intensive Blutdrucksenkung Morbidität und Mortalität zahlreicher Hochrisikogruppen senken kann. Drei Viertel der hypertensiven Patienten mit diabetischer Nephropathie sterben an kardialen oder zerebralen Ursachen. Die Mortalität dieser Patienten wird entscheidend durch koronare Herzerkrankung und Überstimulation des Myokards durch das sympathische Nervensystem infolge der autonomen Neuropathie beeinflusst Eine antihypertensive Betablockertherapie hat einen, über die Blutdrucksenkung hinausgehenden, kardioprotektiven Effekt (7).

Die optimalen Blutdruckwerte sind bisher nicht genau definiert. Die HOT-Studie zeigte, dass bei diabetischen Hypertonikern mit der strengsten Blutdruckeinstellung ( $<80 \mathrm{mmHg}$ diastolisch) die schwerwiegenden kardiovaskulären Ereignisse am geringsten waren (mittlere Beobachtungszeit: 3,8 Jahre) (8). Die Daten des Joint National Committee on Prevention, Detection, Evaluation and Treatment of High Blood Pressure (JNC VI) (1997) empfehlen eine Blutdrucksenkung unter $130 / 85 \mathrm{mmHg}$, und bei einer Proteinurie $>1 \mathrm{~g} /$ Tag sogar unter 125/75 mmHg. Den ACE-Hemmern kommt zwar eine hervorragende Bedeutung in der Senkung des Blutdrucks und der Verminderung der Proteinurie zu, allerdings zeigen aktuelle Daten einer doppelblinden randomisierten Studie, dass auch Dihydropyridin-Kalziumantagonisten die Progression bzgl. der Albuminurie bei normotensiven Patienten mit Typ-1-Diabetes ebensogut vermindern wie die ACEHemmer (9). Ähnliche Befunde bei Typ-2-Diabetikern finden sich auch für den Vergleich von lang wirksamen Dihydropyridin-Kalziumantagonisten und $\beta$-Blockern bzgl. der Progression der Nephropathie (10). Aus Daten der HOPE-Studie wurde allerdings deutlich, dass bei Patienten mit Typ-2-Diabetes unter ACE-Hemmer Therapie (unabhängig vom Blutdruck) signifikant weniger kardiovaskulären Komplikationen auftraten (11).

lkurzgefasst: Bei Patienten mit Diabetes mellitus wird empfohlen, arterielle Blutdruckwerte unter 125/85 mmHg anzustreben. Insbesondere bei älteren und gefäßkranken Patienten ist eine zu rasche Blutdrucksenkung mit der Gefahr kritischer Ischämien, Sturzgefahr etc. behaftet. Auch ältere Patienten profitieren jedoch eindeutig von einer guten, an den Funktionszustand adaptierten antihypertensiven Therapie. Eine ansteigende Mortalität bei „zu niedrigen Blutdruckkurven“ (J-Kurve), scheint es nicht zu geben. Als Mittel der ersten Wahl sind ACE-Hemmer, $\beta$-Blocker, Dihydropyridin-Kalziumantagonisten und Diuretika anzusehen.

\section{ACE-Hemmer}

Bei Typ-1-Diabetikern mit diabetischer Nephropathie wurde in zahlreichen Studien ein positiver Effekt der ACE-Hemmer auf Proteinurie und Filtratabfall gezeigt. Dies gilt auch für normotensive Patienten mit Proteinurie oder Mikroalbuminurie. Prospektive randomisierte Studien, die ACE-Hemmer mit Kalziumantagonisten bzw. $\beta$-Blockern verglichen, konnten bei Typ-2-Diabetikern bezüglich der Verlangsamung des Nierenfunktionsverlustes einen günstigeren Effekt der ACE-Hemmer gegenüber anderen Antihypertensiva nicht einheitlich nachweisen. In der UKPDSStudie konnte für Captopril kein spezieller nephroprotektiver Effekt nachgewiesen werden $(12,13)$. Lediglich für den Surrogatparameter „Albuminurie“ ist eine Überlegenheit der Substanzklasse eindeutig belegt $(22,1)$. Die Wertigkeit der ACE-Hemmer als potente Antihypertensiva ist allerdings unbestritten.

\section{$\mathrm{AT}_{1}$-Rezeptorantagonisten}

Die RENAAL Studie beobachtete 1513 Patienten mit manifester diabetischer Nephropathie (Proteinurie $>0,5 \mathrm{~g} /$ die, mittlerer Kreatininwert $1,9 \mathrm{mg} / \mathrm{dl}$ ) über 3,4 Jahre mit der Frage, ob ein kombinierter Endpunkt aus Tod, Nierenversagen und Verdopplung des Kreatinin-Wertes durch $\mathrm{AT}_{1}$-Rezeptorblocker zu beeinflussen sei. Es fand sich eine Ereignisreduktion von 3,8\%, entsprechend einer „Number Needed to Treat (NNT)“ von 28, d.h. 28 Patienten müssen 3,4 Jahre behandelt werden um ein Ereignis zur verhindern (15). Dabei nimmt man allerdings einen geringen Anstieg der Mortalität in Kauf (wie auch in Elite II und RESOLVD gezeigt). In der IDNT Studie wurden 1715 Patienten mit diabetischer Nephropathie (Proteinurie $>900 \mathrm{mg} / \mathrm{d}$ und Serumkreatininwerte: $1,2-3,0 \mathrm{mg} / \mathrm{dl}$ ) mit Irbesartan versus Amlodipin behandelt. Nach 2,6 Jahren sank die Wahrscheinlichkeit für den kombinierten Endpunkt aus Tod, Nierenversagen und Verdopplung des Kreatininwertes um 6,4\%, ohne dass die Mortalität gesenkt werden konnte (16). In der IRMA-2-Studie erhielten 590 hypertensive Typ-2-Diabetiker mit beginnender diabetischer Nephropathie (Mikroalbuminurie: $20-200 \mu \mathrm{g} / \mathrm{min}$ ) mindestens 2 Jahre lang Irbesartan oder Plazebo. Zusätzlich konnten weitere Antihypertensiva außer ACE-Hemmern und anderen Angiotensin-II-Antagonisten eingesetzt werden, um Blutdruckzielwerte $<135 / 85 \mathrm{mmHg}$ zu erreichen. Primärer Endpunkt war die Entwicklung einer Proteinurie. In der Plazebogruppe entwickelte sich bei 14,9\% der Patienten eine Proteinurie, bei 9,7\% der Patienten mit $150 \mathrm{mg}$ Irbesartan und bei 5,2\% der Patienten mit 300 mg Irbesartan. Das Risiko, eine Proteinurie zu entwickeln, lag damit in der Patientengruppe, die $300 \mathrm{mg}$ Irbesartan erhalten hatte, signifikant um 9,7\% niedriger. Leider macht die Studie keine Angaben, ob Sartane eine Verschlechterung der Nierenfunktion verzögern können (17). Alle drei Studien haben Sartane nicht gegen ACE-Hemmer getestet. Dies ist umso bedauerlicher, da Sartane in der Therapie der häufig bei Diabetikern vorkommenden Herzinsuffizienz tendenziell schlechter abschneiden als ACE-Hemmer. Bei fortgeschrittener Niereninsuffizienz sind $\mathrm{AT}_{1}$-Rezeptorantagonisten noch antihypertensiv und antiproteinurisch wirksam ohne die Nierenfunktion relevant zu beeinträchtigen (23). Sie haben ein günstigeres Nebenwirkungsprofil.

Kombination von $\mathrm{ACE}-H e m m e r n$ und $\mathrm{AT}_{\mathbf{1}}$-Rezeptorblocker Vereinzelte Berichte weisen auf eine mögliche additive Wirkung von ACE-Hemmer und $\mathrm{AT}_{1}$-Rezeptorblocker hin. Die Candesar- 
tan- und Lisinopril-Mikroalbumin Studie (CALM) hat einen günstigen Effekt auf den Blutdruck und die Urinalbuminausscheidung bei dualer Blockade des RA-Systems bei hypertensiven Typ-2-Diabetikern gezeigt (14) Bei dieser Behandlung sollten aufgrund der stark blockierten Aldosteronwirkung und des möglichen Einflusses auf die GFR vor allem das Serumkalium und die Serumkreatininwerte gut überwacht werden. Die kombinierte RA-Systemblockade wird derzeit nicht als Therapie der ersten Wahl empfohlen.

lkurzgefasst: Für Typ-1-Diabetiker mit diabetischer Nephropathie konnte ein nephroprotektiver Effekt von ACEHemmern gezeigt werden - bei Typ-2-Diabetikern waren die Ergebnisse nicht einheitlich. Die Wertigkeit der ACEHemmer als potente Antihypertensiva bleibt jedoch unbestritten. AT $_{1}$-Rezeptorblocker sind auch bei Niereninsuffizienz noch antihypertensiv und antiproteinurisch wirksam. Sie reduzieren das Fortschreiten der diabetischen Nephropathie. Allerdings gibt es keine Studien, in der $\mathrm{AT}_{1}$ - $\mathrm{Re}$ zeptorblocker mit ACE-Hemmern verglichen werden. So bleibt die Wahl zwischen beiden Substanzen bis auf weiteres schwierig. Eine Kombination von ACE-Hemmern und $\mathrm{AT}_{\mathbf{1}}$-Rezeptoblocker wird derzeit nicht als Therapie der ersten Wahl empfohlen.

\section{Redulktion kardiovaskulärer Risilkofalktoren}

Zu Beginn jeder Therapie, insbesondere bei älteren Menschen, sollten die Therapieziele definiert werden. Nach den Richtlinien der Europäischen Arteriosklerose Gesellschaft sind Diabetiker Hochrisikopatienten. Bei gefäßkranken Diabetiker müssen alle kardiovaskulären Risikofaktoren bestmöglich reduziert werden. Das Risiko stiller myokardialer Ischämien und Infarkte ist bei Diabetikern besonders hoch und wird auf bis zu 30\% geschätzt. Da die Mortalität der Typ-1und Typ-2-Diabetiker im Wesentlichen durch das kardiovaskuläre Risiko geprägt ist (Tab.1), sollten frühzeitig nicht-invasive und invasive Techniken zur Diagnostik der koronaren Herzerkrankung eingesetzt werden. Diabetiker erleiden häufig ein akutes Nierenversagen bei Diagnostik und Therapie von diabetischen Begleitkomplikationen (34). Neben der klassischen Koronarangiographie bietet künftig auch die hochauflösende Magnetresonanztomographie Möglichkeiten zur frühzeitigen Detektion von Stenosen der Koronararterien und damit zur sekundären Prävention. Bestehen keine Kontraindikationen, so sollte bei Diabetikern mit hohem kardiovaskulärem Risiko eine Thrombozytenaggregationshemmung durchgeführt, ein Cholesterin-Synthese-Hemmer verordnet und zu Nikotinverzicht (Raucherentwöhnung) geraten werden. Insbesondere die Intensität der Statintherapie sollte nicht nur von der Höhe des aktuellen Serumcholesterinspiegels abhängig gemacht werden, sondern vielmehr vom kardiovaskulärem Gesamtrisiko des Patienten. Zwar ist ein Zusammenhang zwischen erhöhten Cholesterinwerten und Progredienz einer Niereninsuffizienz nicht bewiesen, jedoch ist ein hohes Gesamtcholesterin bei Dialysepatienten auch ein Marker für längeres Überleben. Man geht heute davon aus, dass bei Diabetikern bevorzugt sog. „Small dense LDL-Partikel“ akkumulieren, die rasch oxidieren und ein hohes atherogenes Potenzial haben. Die Wertigkeit einer medikamentösen Senkung des Serumcholesterinspiegels bei Patienten mit Typ-1- oder Typ-2-Diabetes für die Progression der Nephropathie ist noch umstritten und derzeit noch Fragestel-
Tab. 1 Kardiovaskuläres Risikoprofil

$$
\begin{aligned}
& \Rightarrow \text { LV Hypertrophie } \\
& \Rightarrow \text { Koronare Makroangiopathie } \\
& \Rightarrow \text { Koronare Mikroangiopathie }
\end{aligned}
$$

lung größerer Studien (Die Deutsche Diabetes Dialyse Studie 4D, CHORUS, UK-HARP, ESPLANADE).

Erhöhte Homocysteinwerte sind ebenso wie Lipoprotein A ein unabhängiger kardiovaskulärer Risikofaktor in der Allgemeinbevölkerung und bei Dialysepatienten, bei Diabetikern sind sie jedoch nur schwach mit dem Auftreten einer Nephropathie assoziiert. Die Marker für oxidativen Stress sind im Serum von diabeteskranken Hämodialysepatienten ebenfalls erhöht (Malondialdehyd, oxidiertes LDL, Erythrozytenlguthationperoxidase etc.). Die Bedeutung dieser Befunde ist noch unklar. Erhöhte Fibrinogenspiegel sind ein unabhängiger kardiovaskulärer Risikofaktor bei diabetischen Hämodialyse-Patienten. Eine therapeutische Konsequenz ergibt sich aus diesen Befunden bisher noch nicht.

\section{Therapie der chronischen Niereninsuffizienz}

Auf die Bedeutung eines ausgeglichenen Säure-Basenhaushaltes ( $\mathrm{Bi}$ karbonatsubstitution), die Senkung erhöhter Phosphatspiegel durch Kalzium- oder Aluminiumsalze, die Behandlung eines sekundären Hyperparathyreoidismus, ggf. durch Kalzium- oder Vitamin D-Substitution, den Ausgleich von Störungen des Wasser- und Elektrolythaushaltes und die Anhebung des Hämoglobinwertes auf Werte von $12 \mathrm{~g} / \mathrm{dl}$ sind wir bereits im Teil Diagnostik eingegangen.

Harnwegsinfekte treten häufiger bei Diabetikern mit schlechter Stoffwechseleinstellung und Blasenfunktionsstörungen auf. Ein Aufsteigen des Infektes kann zu einer chronischen Entzündung bis hin zu Papillennekrosen führen. Die Diagnostik und Therapie von Blasenentleerungsstörungen sowie Harnwegsinfekten gehört deshalb obligatorisch zur Behandlung von Diabetikern mit Nephropathie.

\section{Dialyse}

Dialysebeginn: Bei fortgeschrittener Niereninsuffizienz sollte die Dialysebehandlung bei diabetischer Nephropathie frühzeitig eingeleitet werden, da die metabolischen Nebenwirkungen der Urämie (Azidose, Katabolie, arteriosklerotische Gefäßerkrankungen) gegenüber nicht diabetischen Patienten verstärkt sind. Es wird empfohlen, ab einer GFR von 15-20 ml/min die Dialysebehandlung zu beginnen (bei nicht Diabetikern ab einer GFR von $10 \mathrm{ml} / \mathrm{min}$ ) (18). Um die GFR adäquat zu bestimmen, können Kreatinin- und Harnstoff-Clearance bestimmt und dann der Mittelwert aus beiden Werten berechnet werden. Individuell kann bei Vorliegen einer schweren Hypertonie, einer Überwässerung oder einer metabolischen Azidose die Dialysetherapie auch früher begonnen werden. Die Auswahl des Therapieverfahrens (Peritoneal- oder Hämodialyse) muss in Anbetracht der individuellen Situation des Patienten nach einer ausreichend umfangreichen Beratung erfolgen. Diese sollte auf jeden Fall schon weit vor dem Auftreten einer Dialyseindikation erfolgt sein. Ein eindeutiger Morbiditäts- oder Mortalitätsvorteil von Hämo- oder Peritonealdialyse hat sich bislang nicht ergeben $(18,19)$. 
Peritonealdialyse: Die Peritonealdialyse ist als Heimdialyseverfahren besonders bei Patienten von Vorteil, die eine selbständige eigenbestimmte Lebensführung wünschen und ggf. die berufliche und soziale Integration mit diesem Verfahren besser erhalten können. Auf hämodynamischer Seite ist die kontinuierliche Dialyse mit geringeren Volumenschwankungen hervorzuheben, die insbesondere bei Patienten mit bestehender autonomer Neuropathie mit Blutdruckschwankungen von Vorteil ist. Die Restnierenfunktion bleibt unter der Peritonealdialyse länger erhalten (20). Bei Patienten mit schwierigem Gefäßzugang, ggf. auf dem Boden einer Makroangiopathie, kann die Peritonealdialyse die einzige Alternative darstellen. Nachteile sind die Entstehung von Infektionen (insbesondere Katheterinfektionen und Peritonitiden), die jedoch gegenüber nicht-diabetischen Dialysepatienten nicht gesteigert sind (24). Die Kontrolle der Blutglukose bei der Peritonealdialyse ist durch entsprechende Anpassung der Insulindosis gleich gut wie bei der Hämodialyse zu gestalten. Die technische Funktionsrate liegt bei der Peritonealdialyse mit einer Halbwertzeit von ca. 4-5 Jahren allerdings niedriger als an der Hämodialyse.

Hämodialyse: Eine hohe Clearanceleistung ist bei großen und schweren Patienten mit der Hämodialyse einfacher zu realisieren. Insbesondere bei Überwässerung und ausgeprägter Hypertonie ist durch die 4-5mal wöchentliche Hämodialyse oder die nächtliche Limited-Care-Dialyse eine Verbesserung der Blutdruckeinstellung zu erzielen (25). Der korrekten Einstellung des Trockengewichtes kommt insbesondere beim Diabetiker eine hohe Bedeutung zu. Dies ist Voraussetzung für eine adäquate Einstellung des Blutdrucks (26). $\mathrm{Zu}$ hohe Ultrafiltrationsraten (>3 1/Dialyse) sollen bei häufig eingeschränkter autonomer nervaler Regulation vermieden werden. Hier ist eine $4 \mathrm{x}$ wöchentliche Hämodialyse oder auch die tägliche Heimdialyse zu erwägen. Die dauerhafte Zugangsmöglichkeit über einen Cimino-Shunt ist bei Diabetikern an der Hämodialyse häufig technisch schwierig, insbesondere bei bestehender Makroangiopathie bzw. Mediasklerose an den Armgefäßen. Die Langzeitfunktion der Shunts ist damit insgesamt schlechter. Eine Handgangrän kommt fast ausschließlich bei diabetischen Dialysepatienten vor.

\section{lkurzgefasst: Bei fortgeschrittener Niereninsuffizienz}

sollte die Dialysebehandlung frühzeitig eingeleitet werden, da metabolische Nebenwirkungen der Urämie gegenüber nicht-diabetischen Patienten verstärkt sind. Die Entscheidung für Peritoneal- oder Hämodialyse richtet sich nach der individuellen Situation des Patienten.

\section{Transplantation}

\section{Nierentransplantation}

Für Diabetiker ist die Nierentransplantation bzgl. Mortalität und Morbidität günstiger als die Dialyse. Die Überlebensrate nach Nierentransplantation liegt bei ca. $60 \%$ für Typ-2-Diabetiker nach 5 Jahren (27). Dagegen beträgt die 5-Jahres-Überlebensrate unter Hämodialyse und Peritonealdialyse bei dieser Patientengruppe weniger als 25\% (28). Die Patientenüberlebensrate von Typ-1-Diabetikern liegt nach alleiniger Nierentransplantation deutlich höher als bei Typ-2-Diabetikern (ca. 86\%) (29). Prä- und Posttransplantationskontrolle sowie der Einsatz der Immunsuppression sind bei Diabetikern und Nicht-Diabetikern grundsätzlich vergleichbar. Bei den Infektionen in der Posttransplantationsphase spielen bei Diabetikern Harnwegsinfektionen eine besondere Rolle und bedürfen der engen Kontrolle (Ursachen: Glucosurie, neurogene Blasenfunktionsstörungen). Aufgrund der hohen Inzidenz von koronaren bzw. peripheren arteriosklerotischen Veränderungen, muss insbesondere in der Prä- aber auch in der Posttransplantationsperiode eine sorgfältige Evaluation bezüglich einer Makroangiopathie durchgeführt werden. Dazu gehören u.a. auch Doppler-Ultraschalluntersuchungen und arterielle DSA-Untersuchungen der Beckenstrombahn (wichtig bezüglich Gefäßanschlusssituation des Transplantates) und der peripheren Beingefäße. Viele Zentren fordern eine Koronarangiographie und die Beseitigung signifikanter Koronarstenosen routinemäßig vor Transplantation. Die nicht-invasiven Diagnoseverfahren (BelastungsEKG, Stress-Echokardiographie, Myokardszintigraphie) haben hier nur eine eingeschränkte Sensitivität. Es hat sich gezeigt, dass auch bei Diabetikern die Prognose des Transplantatüberlebens bei der Lebendspendetransplantation höher ist als bei der Leichenspende (30). Ein Wiederauftreten einer diabetischen Nephropathie im Transplantat ist bei schlechter Blutzuckereinstellung bereits nach ca. 5 Jahren möglich und kann sich in einer großen Proteinurie und Funktionsverschlechterung des Transplantats äußern.

\section{Nieren-Pankreas-Transplantation}

Bei Patienten mit Typ-1-Diabetes kann mit einer kombinierten Nieren-Pankreas-Transplantation nach einigen Studienergebnissen eine weitere Reduktion der Mortalität im Vergleich zur alleinigen Nierentransplantation beobachtet werden (31). Die Einjahresüberlebensrate der Patienten liegt in großen Zentren bei 95\%, die Funktionsrate des Pankreastransplantats bei $85 \%$. Beim dialysepflichtigen Diabetiker ist die simultane Nieren-Pankreas-Transplantation vor allen Dingen dann eine Behandlungsoption, wenn gleichzeitig eine schwer einstellbare Stoffwechselsituation vorliegt oder weitere Komplikationen, z.B. eine schwere Neuropathie, eine Verbesserung der Stoffwechsellage dringlich erscheinen lässt. Patienten mit Doppeltransplantation haben gegenüber nur nierentransplantierten Patienten eine gesteigerte Frühmorbidität (32). Dies betrifft auch die Frühmortalität aufgrund der höheren Komplikationsrate in der postoperativen Phase nach Pankreastransplantation. Hierzu zählen vor allen Dingen die Venenthrombose und Infektionen des Pankreastransplantates. Als Vorteil bei funktionierendem Pankreastransplantat kann v.a. die Verbesserung der Lebensqualität angesehen werden. Auch Verbesserungen bei der Neuropathie, einschließlich der autonomen Neuropathie, wurden gefunden. Es gibt auch Hinweise, dass nach einer erfolgreichen Pankreastransplantation und normoglykämischer Stoffwechselsituation für mindestens fünf Jahre, eine Regression einer diabetische Nephropathie möglich ist. Die diabetische Retinopathie ist kaum rückbildungsfähig, scheint sich aber zu stabilisieren $(21,33)$.

kurzgefasst: Für Diabetiker mit terminaler Niereninsuffizienz ist die Nierentransplantation bezüglich Morbidität und Mortalität günstiger als die Dialyse. Bei Patienten mit Typ1-Diabetes kann durch eine Kombinierte Nieren-Pankreastransplantation die Mortalität im Vergleich zur alleinigen Nierentransplantation noch weiter gesenkt werden. Die simultane Nieren-Pankreas-Transplantation ist vor allem dann eine Option, wenn gleichzeitig eine schwer einstellbare Stoffwechselsituation oder Komplikationen vorliegen, die eine Verbesserung der Stoffwechsellage erfordern. 
Literatur

${ }^{1}$ The Microalbuminuria Captopril Study group. Captopril reduces the risk of nephropathy in IDDM with microalbuminuria. Diabetologia 1996; 39(5): 587-593

2 Brodsky IG, Robbins DC, Hiser E et al. Effects of low-protein diets on protein metabolism in insulin-dependent diabetes mellitus patients with early nephropathy. J Clin Endocrinol Metab 1992; 75: 351-357

3 The Diabetes Control and Complications Research Group. Effect of intensive therapy on the development and progression of diabetic nephropathy in the Diabetes Control and Complications Trial. Kidney Int 1995; 47: 1703-1720

${ }^{4}$ UK Prospektive Diabetes Study Group. Intensive blood glucose control with sulfonylureas or insulin compared with conventional treatment and risk of complications in patients type 2 diabetes (UKPDS 33). Lancet 1998; 352 $837-853$

5 Breyer JA, Bain RP, Evans JK et al. The Collaborative Study Group. Predictors of the progression of renal insufficiency in patients with insulin-dependent diabetes and overt diabetic nephropathy. Kidney Int 1996; 50: 1651-1658

${ }^{6}$ Christensen CK, Krusell LR, Mogensen CE. Increased blood pressure in diabetes: essential hypertension or diabetic nephropathy? Scand J Clin Lab Invest 1987; 47: 363-370

7 Sawicki PT, Dähne R, Bender R et al. Prolonged QT interval as a predictor of mortality in diabetic nephropathy. Diabetologia 1996; 39: 77-81

8 Hansson L, Zanchetti A, Carruthers SG et al. Effects of intensive blood pressure lowering and low dose aspirin in patients with hypertension: principal results of the Hypertension Optimal Treatment (HOT) randomised trial. Lancet 1998; 351(9118): 1755-1762

${ }^{9}$ Crepaldi G, Carta Q Deferrari G et al. Effects of lisinopril and nifedipine on the progression of overt albuminuria in IDDM patients with incipient nephropathy and normal blood pressure. Diabetes Care 1998; 21: 104-110

10 Estacio RO, Jeffers BW, Gifford N, Schrier RW. Effect of blood pressure control on diabetic microvascular complications in patients with hypertension and type 2 diabetes. Diabetes Care 2000; 23: B54-B64

11 HOPE study investigators: Effects of ramipril on cardiovascular and microvascular outcomes in people with diabetes mellitus: Results of the HOPE study and MICRO-HOPE substudy. Lancet 2000; 355: 253-259

12 UK Prospektive Diabetes Study Group. Efficacy of atenolol and captopril in reducing risk of macrovascular and microvascular complications in Type 2 diabetes (UKPDS 39). Brit Med J 1998; 317: 713-720

13 Maschio G, Alberti D, Janin G. The effect of the angiotensin converting enzyme inhibitor benazepril on the progression of chronic renal insufficiency. N Engl J Med 1996; 334: 939-945

${ }^{14}$ Mogensen . Role of dual blockade of the renin angiotensin system in hypertensive, microalbuminuric, non insulin dependent diabetes: The Calm Study. Brit Med J 2000; 321: 1440-1444

15 Brenner BM, Cooper ME, de Zeeuw D et al. Effects of losartan on renal and cardiovascular outcomes in patients with type 2 diabetes and nephropathy. N Engl J Med 2001; 345: 861-869
16 NKF-K/DOQI. American Journal of Kidney Dis 2000; 37 (Suppl 1): 1-238

17 Passadakis P, Thodis E, Vargemezis V. Long term survival with peritoneal dialysis in ESRD due to diabetes. Clin Nephrol 2001; 56: 257-270

18 Alloatti S, Manes M, Paternoster G. Peritoneal dialysis compared with hemodialysis in the treatment of end-stage renal disease. J Nephrol 2000; 13: 331-342

19 Ramsay RC, Goetz FC, Sutherland DE. Progression of diabetic retinopathy after pancreas transplantation for insulin-dependent diabetes mellitus. N Engl J Med 1988; 318: 208

20 LewisEJ , Hunsicker LG, Brain RP, Rohde RD. The collaborative study group. The effect of angiotensin-converting-enzyme inhibition on diabetic nephropathy. N Engl. J Med 1993; 329: 1456-62

21 Plum J, Bunten B, Nemeth R, Grabensee B. Effects of the angiotensin Il antagonist Valsartan on blood pressure, proteinuria and renal hemodynamics in patients with chronic renal failure and hypertension. J Am Soc Nephrol 1998; 9: 2223-2234

22 Kraus ES, Spector DA. Characteristics and sequelae of peritonitis in diabetes and nondiabetics receiving chronic intermittent peritoneal dialysis. Medicine 1983; 62: 52-57

23 Pierratos A, Ouwendyk M, Francoeur R, Vas S, Raj DS, Ecclestone AM, Langos V, Uldall R. Nocturnal hemodialysis: three years experience. J Am Soc Nephrol 1998; 9: 859-868

24 Plum J, Schoenicke G, Kleophas W et al. Comparison of body fluid distribution between chronic hemodialysis and peritoneal dialysis patients as assessed by biophysical and biochemical methods. Nephrol Dial Transplant 2001; 16: 2378-2385

25 Kronson JW, Gillingham KJ, Sutherland DE, Matas AJ. Renal transplantation for type II diabetic patients compared with type I diabetic patients and patients over 50 years old: a single center experience. Clin Transplant 2000; 14: 226-234

26 Sitter T, Krautz B, Held E, Schiffl H. Patientenüberleben, Methodenwechsel und Hospitalisierung bei CAPD und Hämodialyse. Dtsch Med Wochenschr 1997; 122: 109-115

$27 \mathrm{Kim} \mathrm{H}$, Cheigh JS. Kidney transplantation in patients with Type I diabetes mellitus: long-term prognosis for patients and grafts. Korean J Intern Med 2001 16: 98-104

28 Terasaki PI. The HLA matching effect in different cohorts of kidney transplant recipients. Clin Transpl 2000: 497-514

29 Smets YF, Westendorp RG, van der Pijl JW et al. Effect of simultaneous pancreas-kidney transplantation on mortality of patients with type I diabetes mellitus and end-stage renal failure. Lancet 1999; 353: 1915-1919

30 Schulak JA, Henry ML, Munda R et al. Pancreas transplantation in Ohio: a 15 year outcomes analysis. Surgery 2001; 130: 546-552

31 Chow VC, Pai RP, Chapman JR, O'Connell PJ, Allen RD, Mitchell P, Nankivell BJ. Diabetic retinopathy after combined kidney-pancreas transplantation. Clin Transplant 1999; 13(4): 356-362

32 Schwenger, V.C. Müssig, O. Hergesell et al. Inzidenz und klinische Charakteristika von Niereninsuffizienz bei Diabetikern. Dtsch Med Wochenschr 2001; 126: 1322-1326 